This item was submitted to Loughborough's Institutional Repository (https://dspace.lboro.ac.uk/) by the author and is made available under the following Creative Commons Licence conditions.

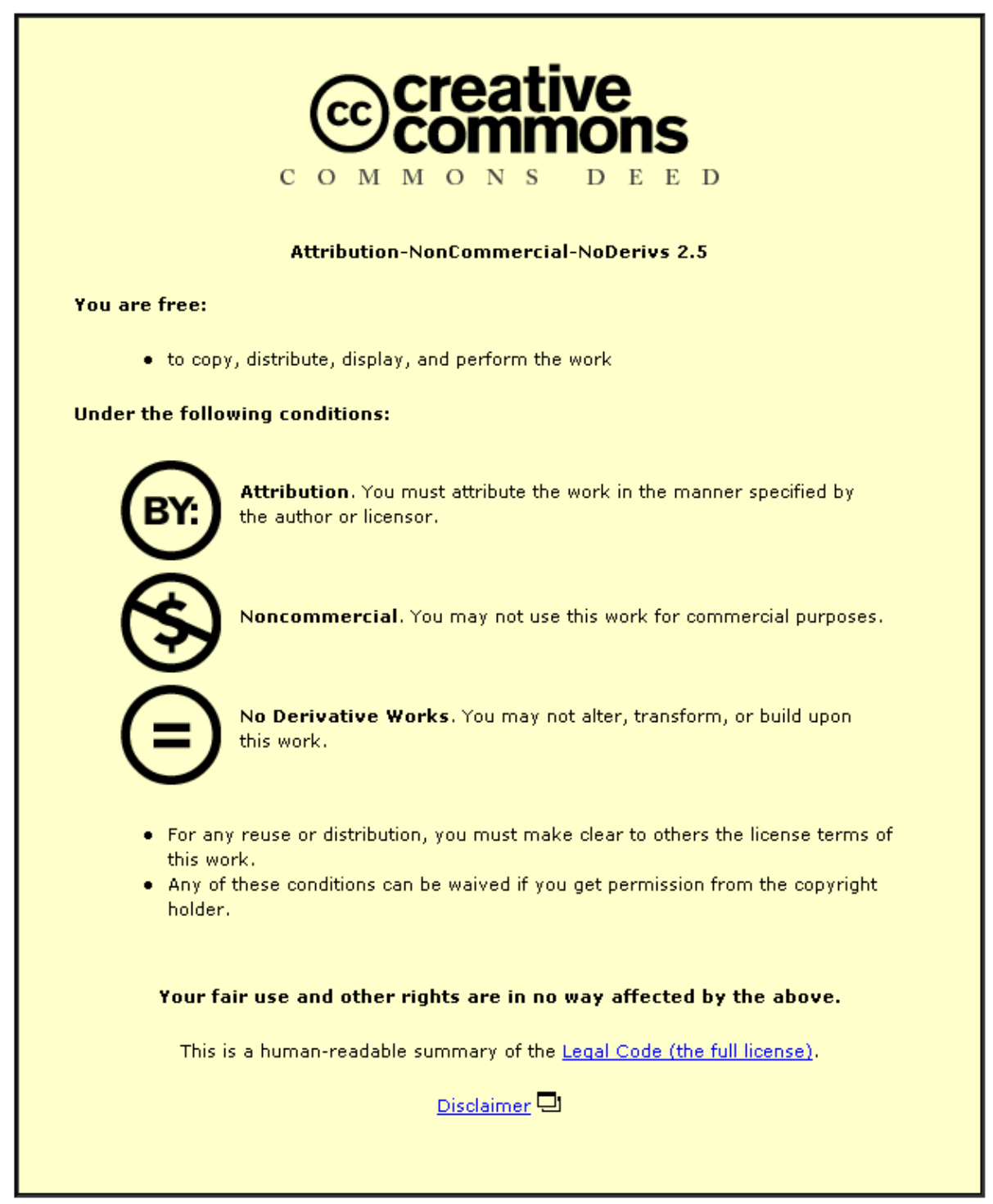

For the full text of this licence, please go to: http://creativecommons.org/licenses/by-nc-nd/2.5/ 


\title{
Laser Vibrometry and the secret life of speckle patterns
}

\author{
Peter Martin \& Steve Rothberg \\ Loughborough University
}

\begin{abstract}
Speckle patterns are produced when coherent light scatters from optically rough surfaces. In typical applications, a Laser Vibrometer collects a region of a speckle pattern on its photodetector. However, noise can be generated in the Laser Vibrometer output when surface motions cause changes in the speckle pattern. This paper uses high resolution images to investigate typical intensity profiles in the partially- and fully-developed speckle patterns scattered from target surfaces with roughnesses in the range $11 \mathrm{~nm}$ to $1 \mu \mathrm{m} \mathrm{Ra}(\approx 1 / 60 \lambda$ to $1.6 \lambda)$ and from a surface treated with retro-reflective tape. Inplane and tilt target motions cause changes in the scattered speckle patterns and sequences of images are used to investigate how the speckle patterns behave in response to these motions. In the case of retro-reflective tape, incident beam diameter is also found to have an important effect on speckle pattern changes and different incident beam diameters are considered for other target surfaces and motions too. This qualitative analysis of the speckle pattern intensity profiles is compared with established theoretical understanding of speckle patterns and their motions and related directly to the instrument noise levels ultimately encountered. Such fundamental evaluation of speckle pattern motions provides a greater appreciation of speckle noise generation in the Laser Vibrometer outputs.
\end{abstract}

\section{Keywords}

Laser Vibrometry; laser speckle; speckle noise; speckle motions; vibration measurement

\section{INTRODUCTION}

A Laser Vibrometer measures the velocity of a moving target using a coherent laser beam. Illuminating the target with the laser beam causes the scattered light to Doppler shift proportionally with velocity of the target in the direction of the beam. The Vibrometer collects a region of the scattered light and mixes it with a coherent reference beam. Frequency demodulation of the Doppler component of the resultant intensity yields the time dependent surface velocity. Micro-scale deviations on the target surface can cause the component wavelets of the scattered light to vary in phase. Subsequently, the scattered, but still coherent, light interferes constructively and destructively as it propagates through space, producing an interference pattern known as a speckle pattern. Target surface motions can cause variations to the scatter, either due to illumination of new surface features or alteration to the surface normal, ultimately causing the sampled speckle pattern to change. Therefore, frequency demodulation of the Doppler component of the resulting intensity combines the changes in phase due to the speckle pattern motions together with the changes in frequency caused by the surface velocity. This additional component to the measured velocity is indistinguishable from genuine surface velocities and is known as speckle noise.

This paper presents initial observations of speckle patterns and speckle motions for a variety of surface finishes, including roughness levels ranging from $\mathrm{Ra} 11 \mathrm{~nm}(\approx \lambda / 60)$ to $1 \mu \mathrm{m}(\approx 1.6 \lambda)$ and a surface treated with retro-reflective tape, with two Vibrometers producing beam spot diameters of $100 \mu \mathrm{m}$ and $600 \mu \mathrm{m}$. Speckle patterns are described as translating or evolving, or some combination of these two behaviours. Any change of the speckle pattern can ultimately add speckle noise to the measured velocity. Using high resolution sequential images, this paper observes the changes to the intensity distributions of speckle patterns qualitatively for in-plane and tilt surface motions and proposes methods to quantify these speckle motions, ultimately attempting to relate these to Laser Vibrometer noise levels. Cross-correlation of these sequential images is used as a tool to observe the speckle pattern changes quantitatively. 


\section{SPECKLE PATTERNS}

\section{Beam spot diameter $=600 \mu \mathrm{m}$}

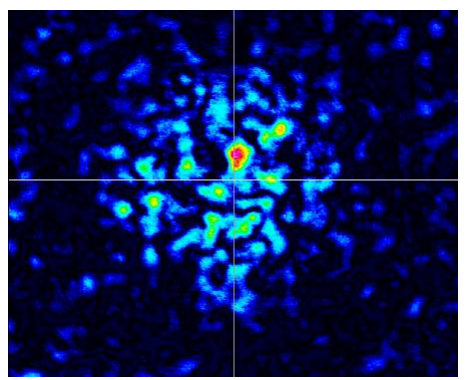

(a)

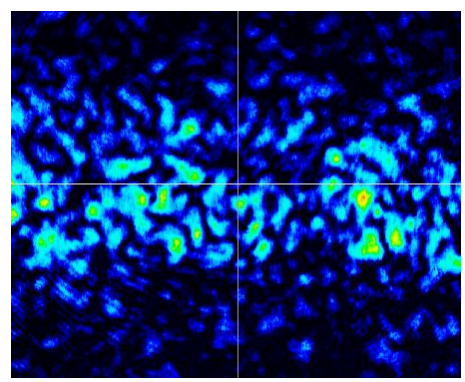

(c)

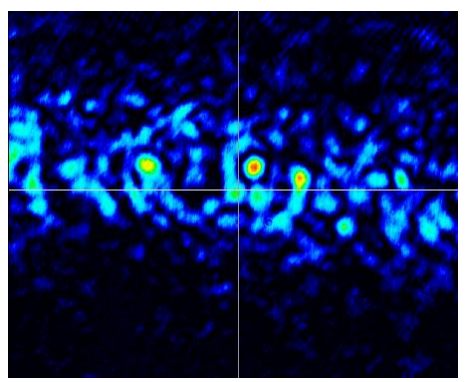

(e)

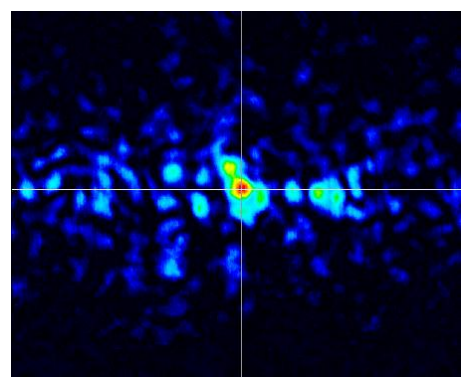

(g)

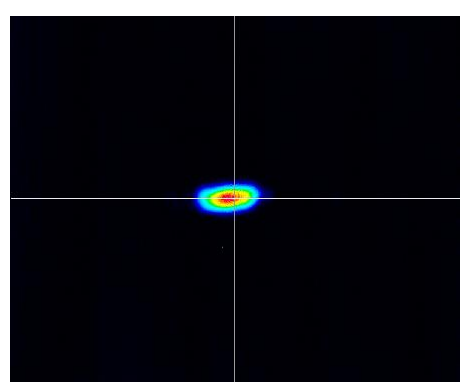

(i)
Retro-reflective tape

Ra $1.0 \mu \mathrm{m}$

Ra $0.37 \mu \mathrm{m}$

Ra 77nm

Ra $11 \mathrm{~nm}$
Beam spot diameter $=100 \mu \mathrm{m}$

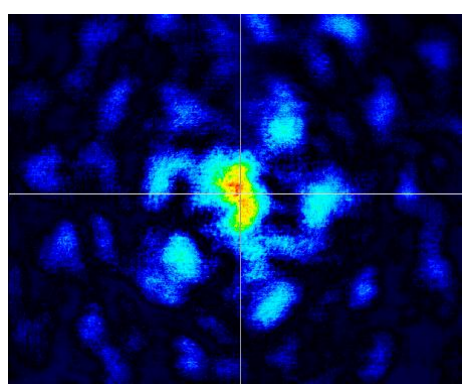

(b)

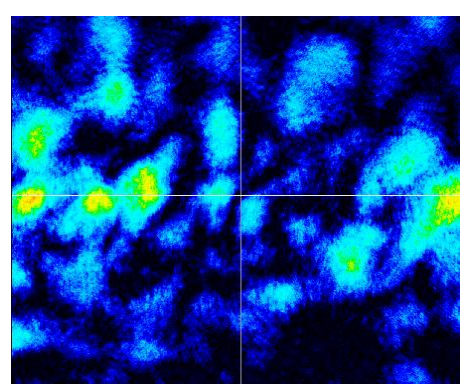

(d)

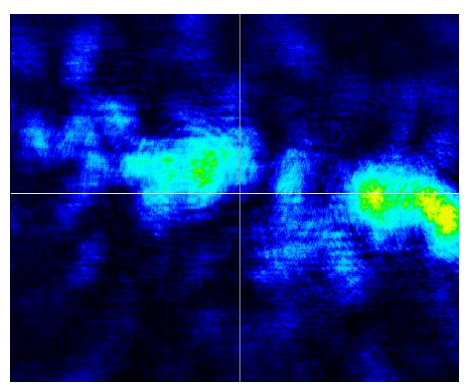

(f)

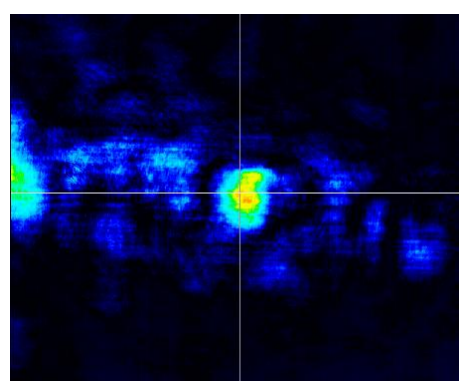

(h)

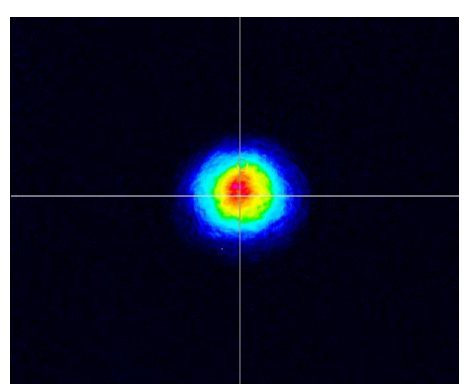

(j)

Figure 2.1: Speckle patterns from a variety of surfaces using beam spot diameters of $600 \mu \mathrm{m}$ and $100 \mu \mathrm{m}$ 
Speckle patterns produced in the scatter from surfaces with deviations comparable or exceeding the wavelength of the light, produce phase excursions in the range of 0 to $2 \pi^{[1]}$ and are considered to be fully developed. Scatter from smoother surfaces likely reduces the range of phase excursions, producing partially developed speckle patterns which might also include features resembling specular reflection. Rough surfaces can significantly reduce the intensity in the scattered light but smooth surfaces can cause unusable scattering angles. Circumstances permitting, retro-reflective tape is commonly used to combat these issues, by maximising the intensity of the scattered light back along the line of the incident beam. Retro-reflective tape produces an intensity pattern in which two intensity distributions are evident. The retro-reflective optics produce an overall Airy disc intensity distribution while the micro-scale deviations in the depth of the surface produce the speckle pattern. Figures 2.1(a)-(j) show typical intensity distributions from surfaces treated with retro-reflective tape and a range of surface roughness producing fully and partially developed speckle patterns which are to be examined in this paper.

The speckle pattern formed with the smaller beam diameter of $100 \mu \mathrm{m}$ and retro-reflective tape, Figure 2.1(b), demonstrates an obvious regularity. The retro-reflective tape is coated in glass beads (approximately 50 $\mu \mathrm{m}$ diameter) embedded into the surface of the tape and separated by gaps similar to the bead diameter. A beam spot diameter of $100 \mu \mathrm{m}$ therefore scatters from only a few glass beads and interference occurs between a small number of phase-varied Airy disc intensity distributions, resulting in the degree of regularity observed in the intensity pattern. A progression from the fully developed speckle patterns produced from the roughest surfaces (Ra $1.0 \mu \mathrm{m}$ and Ra $0.37 \mu \mathrm{m})$ through the combination of partially developed speckle pattern and specular reflection (Ra $77 \mathrm{~nm}$ ) to the specular reflection produced from the smooth surface ( $\mathrm{Ra} 11 \mathrm{~nm})$ can be observed in the figures. Further observation of these images shows a distinct effect of orientation to the scatter from the surfaces without tape. This is a consequence of the features on the surface produced by the machining direction. The surface finish is dominated by corrugations parallel with the machine finish direction. Consequently, the beam scatters more prominently perpendicular to this direction producing the effect shown. This can be observed for the range of surface roughness finishes Ra $1.0 \mu \mathrm{m}$ to $77 \mathrm{~nm}$ (Figures 2.1(c)-(h)). The Ra $11 \mathrm{~nm}$ surface produces an intensity distribution comparable with a mirror-like reflection. This roughness is approximately $\lambda / 60$ (He-Ne $\lambda=632.8 \mathrm{~nm}$ ).

\subsection{Speckle size}

Statistically, the average speckle size, $\left\langle\sigma_{0}\right\rangle$, in a fully developed speckle pattern, defined by the exp[-2] width of the spatial auto-correlation function ${ }^{[2,3]}$ is given by:

$$
\left\langle\sigma_{0}\right\rangle=\frac{2 \sqrt{2} \lambda z}{\pi D}
$$

where $\lambda$ is laser wavelength, $z$ is target surface to observation plane separation distance and $D$ is the Gaussian beam spot diameter by intensity defined by the exp[-2] width. Using the images of the intensity patterns, the average correlation width in the image can be estimated from the auto-correlation function. A two-dimensional auto-correlation can be produced, either directly or by applying the two-dimensional Weiner-Khinchin theorem ${ }^{[1,4]}$. The Weiner-Khinchin method has proven to be a computationally less intensive operation, providing the auto-correlation function through the inverse Fourier transform of the power spectral density. The exp[-2] width of a two-dimensional Gaussian fit to the autocorrelation function is measured producing the average correlation width in the image. The contrast of the intensity distribution, calculated as the ratio of the standard deviation to the average of the intensity ${ }^{[5]}$, can be used as an indication of how well developed the speckle pattern is. For a fully developed speckle pattern, the contrast is unity.

Table 1 shows predicted speckle sizes, which are calculated using Eq. (1), the measured correlation widths $\left(\sigma_{\mathrm{x}}, \sigma_{\mathrm{y}}\right)$ and the contrast for the intensity patterns shown in Figures 2.1. As expected, speckles are larger for the reduced beam spot diameter. The measured correlation widths are all larger than those predicted by classical statistics for fully developed speckle patterns. The contrast of the speckle pattern produced by the surface with roughness Ra $0.37 \mu \mathrm{m}(\approx 0.6 \lambda)$ produces a speckle pattern which is statistically comparable with the speckle pattern developed by the surface with roughness $(\approx 1.6 \lambda)$. The mirror-like reflection produced by the Ra $11 \mathrm{~nm}$ surface produces a contrast far removed from that of a fully developed speckle pattern. As expected, the combination of specular reflection and a speckle pattern observed in Figures 2.1 (g) \& (h) produced by the $\mathrm{Ra} 77 \mathrm{~nm}$ surface produces contrast results suggesting they are partially developed. 
Interestingly, the contrasts calculated for the speckle patterns produced when using retro-reflective tape, for both beam spot diameters, are comparable with the contrasts produced by a smooth surface with $\mathrm{Ra} 77 \mathrm{~nm}$. However, classical speckle statistics do not include the superposition of Airy disc intensity distributions, additional to speckle patterns produced by retro-reflective tape, and therefore it is not surprising to see that they do not conform. Measurement and observation of the speckle size can be related to measured speckle noise levels. Measurements of speckle noise ${ }^{[6]}$ have shown that, for a tilting surface, using a smaller beam diameter can reduce levels of noise. Increasing the speckle size reduces the rate of change of phase caused by the speckle motion, reducing speckle noise measured by the Vibrometer.

\begin{tabular}{|c|c|c|c|c|c|}
\hline $\begin{array}{l}\text { Speckle pattern } \\
\text { surface }\end{array}$ & $\begin{array}{l}\text { Figure } 2.1 . \\
\text { reference }\end{array}$ & $\begin{array}{l}\text { Expected speckle } \\
\text { size, (eqn. }(1), \mu \mathrm{m})\end{array}$ & $\begin{array}{c}\text { Measured } \\
\text { correlation width } \\
\left(\sigma_{\mathrm{x}}, \mu \mathrm{m}\right) \\
\end{array}$ & $\begin{array}{c}\text { Measured } \\
\text { correlation width } \\
\left(\sigma_{\mathrm{y}}, \mu \mathrm{m}\right) \\
\end{array}$ & Contrast \\
\hline Retro-reflective tape & (a) & \multirow{5}{*}{$\begin{array}{c}204 \\
(\mathrm{D}=600 \mu \mathrm{m})\end{array}$} & 418 & 498 & 1.36 \\
\hline $\mathrm{Ra} 1.0 \mu \mathrm{m}$ & (c) & & 301 & 350 & 0.94 \\
\hline $\operatorname{Ra} 0.37 \mu \mathrm{m}$ & (e) & & 341 & 440 & 0.89 \\
\hline $\mathrm{Ra} 77 \mathrm{~nm}$ & $(\mathrm{~g})$ & & 559 & 696 & 1.59 \\
\hline $\mathrm{Ra} 11 \mathrm{~nm}$ & (i) & & 982 & 427 & 3.97 \\
\hline Retro-reflective tape & (b) & \multirow{5}{*}{$\begin{array}{c}808 \\
(\mathrm{D}=100 \mu \mathrm{m})\end{array}$} & 900 & 1343 & 1.32 \\
\hline $\mathrm{Ra} 1.0 \mu \mathrm{m}$ & (d) & & 870 & 1010 & 0.84 \\
\hline $\operatorname{Ra} 0.37 \mu \mathrm{m}$ & (f) & & 1769 & 1164 & 0.84 \\
\hline $\mathrm{Ra} 77 \mathrm{~nm}$ & (h) & & 843 & 1083 & 1.34 \\
\hline $\mathrm{Ra} 11 \mathrm{~nm}$ & (j) & & 1385 & 1285 & 2.87 \\
\hline
\end{tabular}

Table 1. Expected speckle sizes, measured speckle sizes and contrasts of speckle patterns

\section{QUALITATIVE OBSERVATION OF SPECKLE BEHAVIOUR}

Speckles are described as translating or evolving (or 'boiling') and can exhibit both behaviours. In this section, the behaviour of speckles produced from in-plane and tilt target surface motions are to be examined. Incremental in-plane and tilt target surface displacements are produced using displacement stages with resolutions of $10 \mu \mathrm{m}$ and $0.1^{\circ}$ respectively, and sequential images (1200x1024 pixels, resolution of $6.45 \mu \mathrm{m}$ square) are generated.

\subsection{Speckle translation}

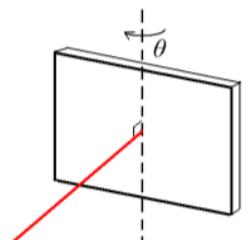

Figure 3.1: Beam alignment on tilting surface

Illumination of a surface which is tilting produces a motion in the intensity patterns which is dominated by translation. For this study, the test surfaces were set in a plane containing the axis of rotation and the beam was aligned to illuminate the surface on the rotational axis as shown in Figure 3.1. For a tilting surface, the speckles are predicted to translate on the observation plane by amount $2 \theta z$ where $\theta$ is the angular displacement (rads) ${ }^{[7]}$

A region of the pattern on the CCD is used later in the quantitative analysis, described in Section 4. The width of the region is chosen to be comparable with the region incident on the Vibrometer's collecting aperture, which requires scaling according to the target to CCD separation and a typical target to Vibrometer separation. This provides the opportunity to relate observed speckle behaviours to the speckle noise levels previously observed ${ }^{[6]}$. The angular increment of the surface $\left(0.1^{\circ}\right)$ would produce speckle translation distance on the CCD of $0.75 \mathrm{~mm}$ and $0.5 \mathrm{~mm}$ for the beam spot diameters of $600 \mu \mathrm{m}$ and $100 \mu \mathrm{m}$ respectively. Figure 3.2 shows example images of speckle patterns and sequential plots of sectional profiles in the direction of the speckle motion. 
(a)

(i)

$0.0^{\circ}$

(ii)

$0.0^{\circ}$

(iii)

$0.1^{\circ}$

(iv)

(v)

(vi)

$0.4^{\circ}$
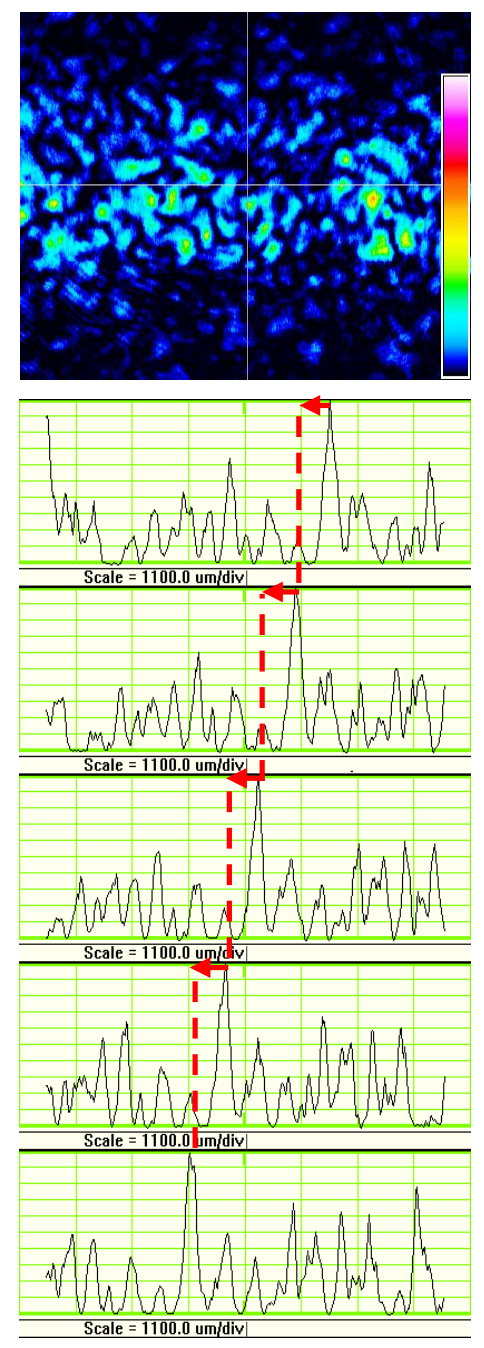

Ra $1.0 \mu \mathrm{m},(D=600 \mu \mathrm{m})$ (b)
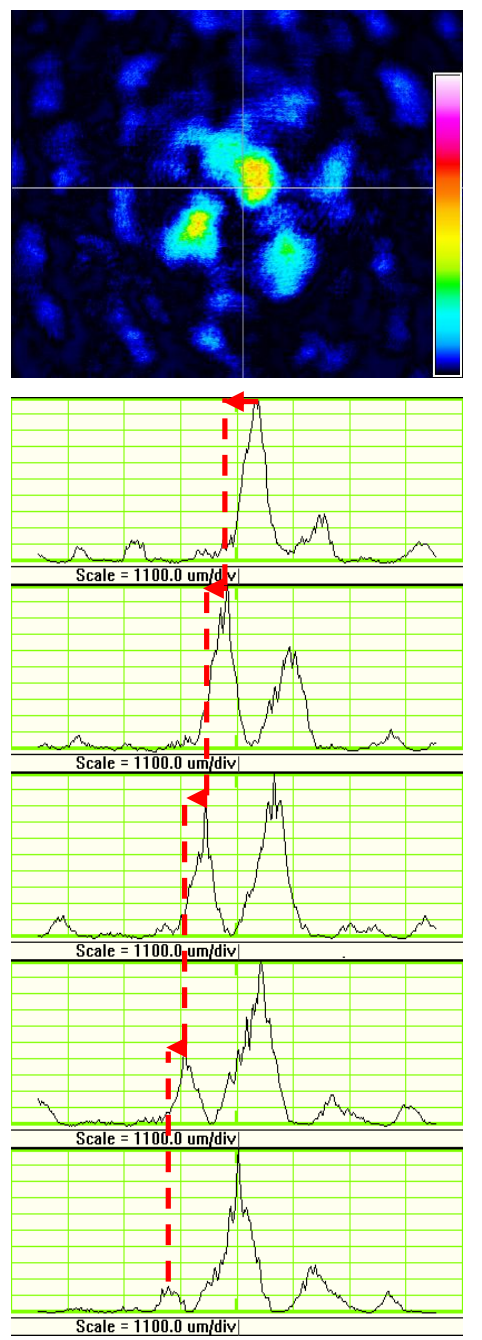

Figure 3.2.

Retro-reflective tape $(D=100 \mu \mathrm{m})$ (c)
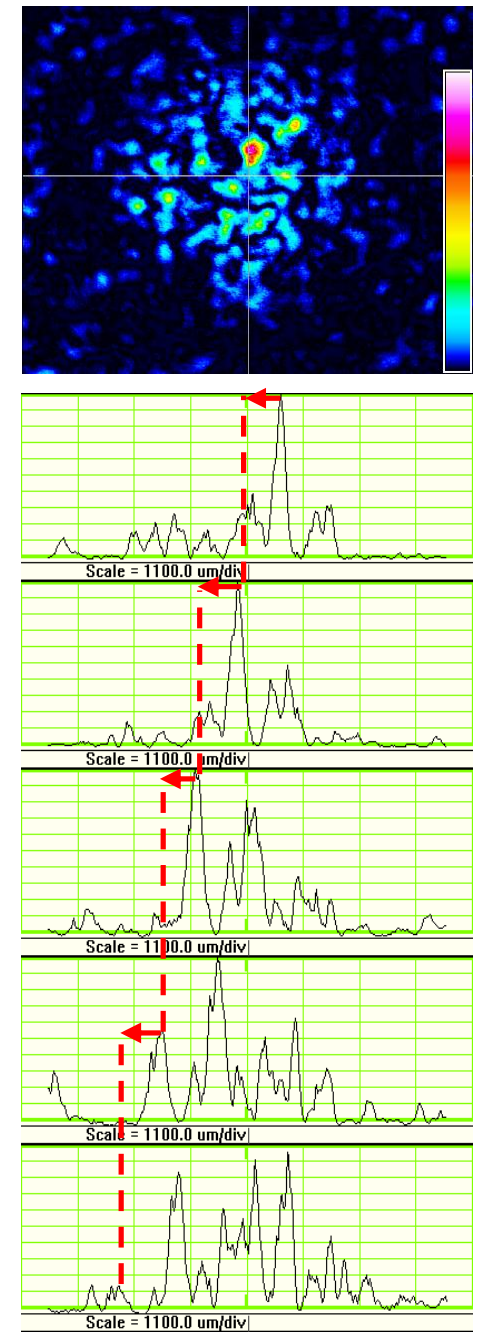

Retro-reflective tape $(D=600 \mu \mathrm{m})$

Figure 3.2 shows example speckle patterns and corresponding sectional profiles as the surface is angularly displaced over $0.4 \mathrm{deg}$ in $0.1 \mathrm{deg}$ increments, causing the speckle pattern to translate from the right to the left in the images. An

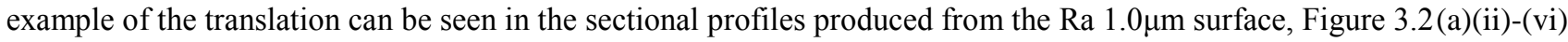
which highlights the same bright speckle in each sequential profile emphasising the direction of the speckle translational motion with the arrows. The speckles do not significantly alter in amplitude or shape and the speckles are considered to be dominated by the translational motion. At this point it is worth noting that as the surface tilts, relative to the beam, the beam spot elongates and becomes elliptical. This illuminates new surface features and will modify the speckle pattern, but the effects of this appear insignificant for the angular scales observed. Speckle translation also exists in the speckle motions when using retro-reflective tape. However, the Airy disc intensity distribution causes the intensity of the speckles to alter as they translate. This is clearer in the sectional profiles of the transitions when using the smaller beam diameter of $100 \mu \mathrm{m}$, where the speckles are relatively large, Figures 3.2(b). A speckle identifiable in Figure 3.2(b)(ii) as having the greatest intensity can be seen to translate, in Figures 3.2(b)(ii)-(vi), as the angular displacement of the surface increases. However, its intensity diminishes as it translates and approaches the first minima of the Airy profile. Concurrently, a second speckle in the speckle pattern, with a significantly lower intensity in Figure 3.2(b)(ii) also translates across the Airy profile but its intensity increases. This transition can also be seen in the sectional profiles in Figures 3.2(c) from speckle patterns produced from the increased beam spot diameter of $600 \mu \mathrm{m}$ and retro-reflective tape. 


\subsection{Speckle Evolution}

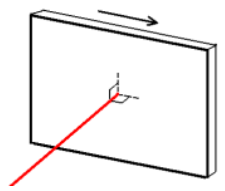

Figure 3.3: Beam alignment on surface translating in-plane

Speckle evolution occurs when there is a change to the population of surface features causing scatter of the beam. This can be the dominant speckle behaviour when the surface translates in-plane i.e. perpendicular to the direction of the beam. This in-plane surface motion can be problematic in causing speckle noise. For this study, the beam is aligned nominally parallel with the surface normal as shown in Figure 3.3. Figures 3.4(a)-(f) show speckle patterns and their corresponding sectional profiles when a surface with Ra $1.0 \mu \mathrm{m}$ is translated in-plane, with increments of $1 / 5$ of the beam diameter, over a total range of one beam spot diameter $(100 \mu \mathrm{m})$.

(a)

$0.00 \mathrm{~mm}$

(b)

$0.02 \mathrm{~mm}$

(c)

$0.04 \mathrm{~mm}$

(d)

$0.06 \mathrm{~mm}$

(e)

$0.08 \mathrm{~mm}$

(f)

$0.10 \mathrm{~mm}$

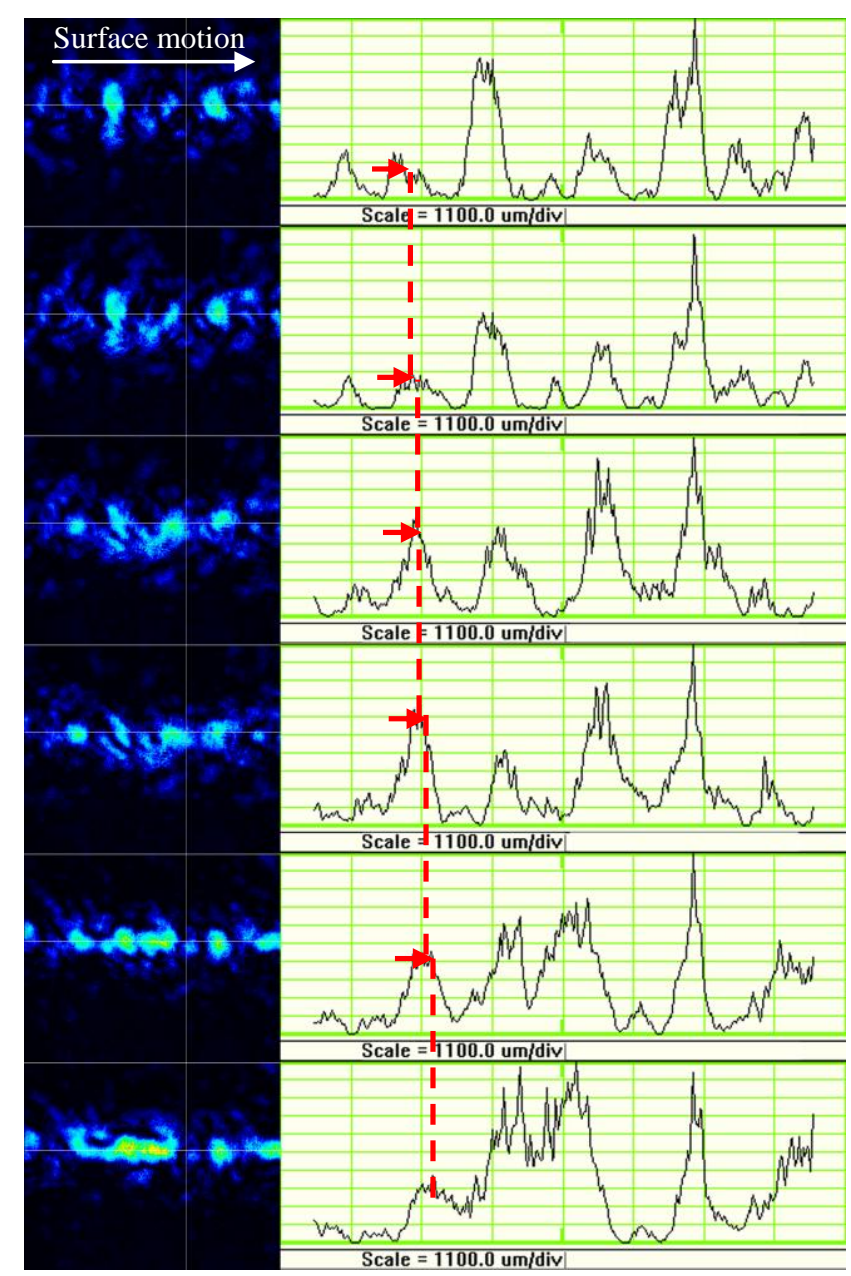

Figure 3.4.

Ra $1.0 \mu \mathrm{m}(D=100 \mu \mathrm{m})$ (a)

$0.00 \mathrm{~mm}$

(b)

$0.12 \mathrm{~mm}$

(c)

$0.24 \mathrm{~mm}$

(d)

$0.36 \mathrm{~mm}$

(e)

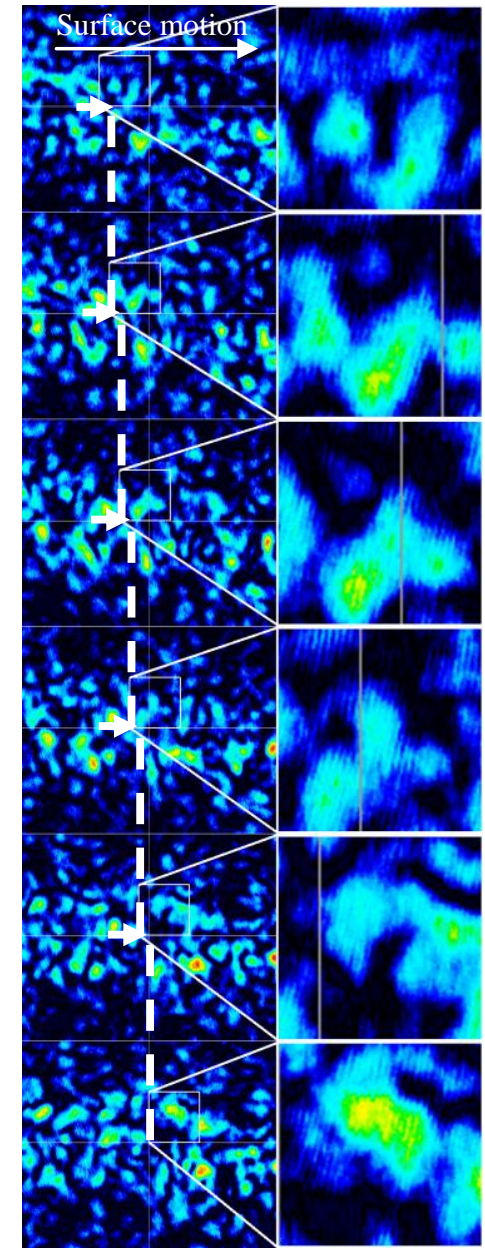

Figure 3.5.

Ra $1.0 \mu \mathrm{m}(D=600 \mu \mathrm{m})$

Figures 3.4(a)-(f) show a change to the intensity of the speckles as well as a slight translation, highlighted by the arrows and vertical lines on the sectional profiles. This change in intensity and shape is the result of the change to the population of surface scatterers and the speckles appear to translate in sympathy with the surface motion. However, the translational behaviour of the speckles is not consistent as described later in Section 4.1. Figures 3.5(a)-(f) present evolution of a region of the speckle pattern from a surface with $\mathrm{Ra} 1.0 \mu \mathrm{m}$ as the surface is displaced $1 / 5$ of beam spot diameter 
$(D=600 \mu \mathrm{m})$. A region of the speckle pattern is tracked at the same increment as the surface displacement and enlarged in the figure. This largely eliminates the effect of translation in the images and concentrates attention solely on the evolution of the speckle pattern. The speckles can be seen to alter in shape and intensity as the surface moves. From the images, the speckles appear to have decorrelated after the surface has translated by $0.36 \mathrm{~mm}$. For specific configurations, it is predicted that a surface can translate up to a maximum of $D / \sqrt{2}{ }^{[2]}$, by which point the speckles will always be uncorrelated. The decorrelation of the speckle pattern is dependent on the surface motion as a proportion of the beam diameter. For a larger beam diameter, the surface is likely to translate further before the correlation is lost and this has been shown to reduce the influence of speckle noise ${ }^{[6]}$. Quantitative analysis of this speckle behaviour would provide a useful description of this motion and this is the subject of the next section.

\section{QUANTITATIVE ANALYSIS OF SPECKLE MOTIONS}

Quantification of speckle behaviours would facilitate a valuable analysis of the mechanisms behind speckle noise. It is proposed to use cross-correlations of the images, in the nominal direction of the speckle translational motion, to quantify the amount of translation and evolution of the speckles. The cross-correlations are performed over a region which is comparable with that collected on the Vibrometer aperture. The changes in intensity, ultimately, are to be related to speckle noise levels but speckle noise is generated principally by phase, which is not easily observed. This analysis relies on the relationship between the measured intensity and its phase, in that the mechanism that causes a change to the intensity will also drive a change in phase.

\subsection{Cross-correlation analysis}

Sequential images of speckle patterns produced from incrementally displacing the surfaces are used to quantify the translation and the evolution of the speckles. An example of the process used is shown in Figures 4.1 to 4.3 using a sequence of speckle patterns produced from a tilting surface which is treated with retro-reflective tape and illuminated with a beam spot diameter of $600 \mu \mathrm{m}$. A region of the speckle pattern, comparable (in the direction of the speckle motion) to that collected on the Vibrometer aperture and located centrally in the initial image, see Figure 4.1(a), is selected as the interrogation region. This region is cross-correlated with the entire image as shown in Figures 4.1(b) and (c) and a normalised auto-correlation, shown in Figure 4.1(d), is produced.

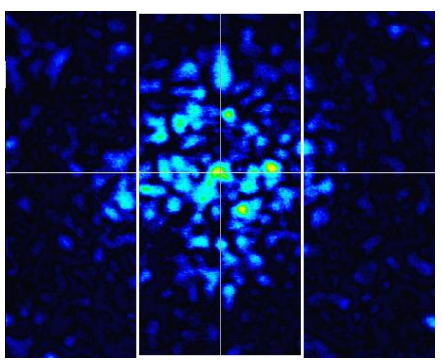

(a)

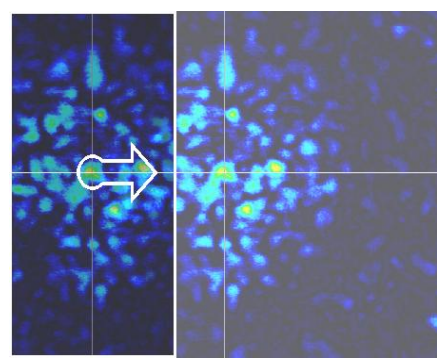

(b)

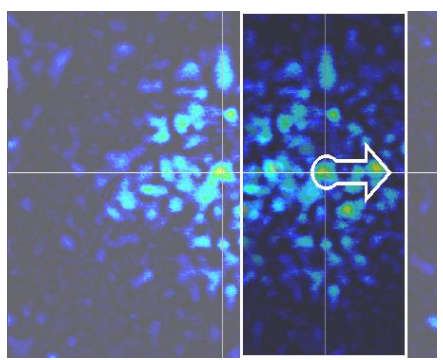

(c)

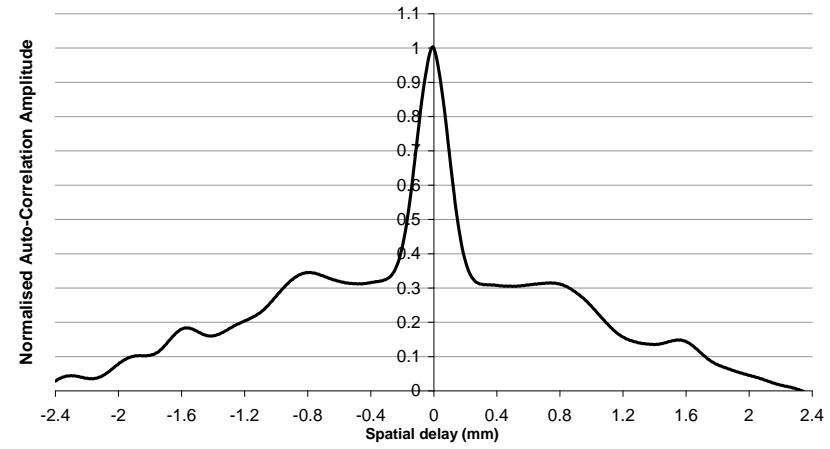

(d)

Figure 4.1: Normalised auto-correlation of surface treated with retro-reflective tape $\left(0.0^{\circ}\right)$ 
This interrogation region, from the initial image, is then cross-correlated with sequential speckle pattern images produced from the incremental angular displacements, see Figures 4.2, which are normalised against the peak of the auto-correlation and presented in Figure 4.3 as a function of delay expressed in terms of aperture size. The average peak spatial delays and corresponding cross-correlation amplitudes can be used to quantify speckle translations and the degree of evolution respectively. A pure speckle translation produces a spatial delay of the peak of the normalised crosscorrelations without change in the amplitude. Pure evolution produces no spatial delay to the peak of the normalised cross-correlations and only a change to the amplitude. A combination of the two speckle motions will produce not only a spatial delay but also a change to the amplitude of the peak of the normalised cross-correlation. However consideration needs to be given to the change in normalised cross-correlation amplitude due to the effects of the Airy disc intensity distribution when using the retro-reflective tape on a surface which is tilting.
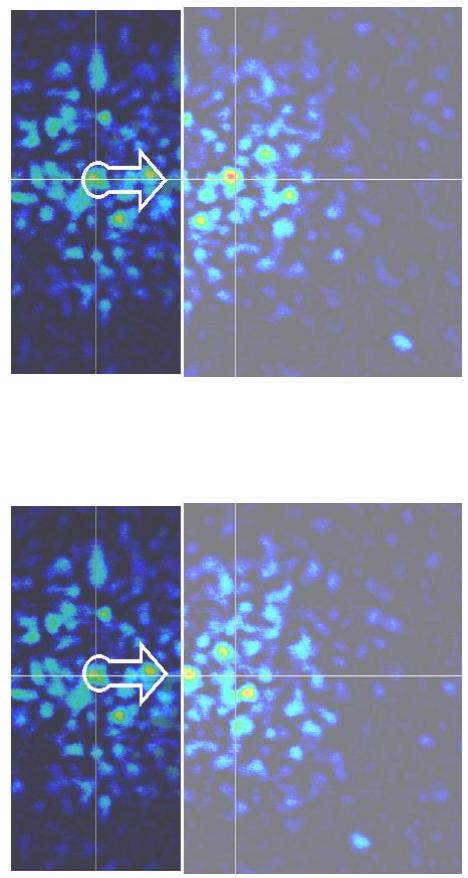

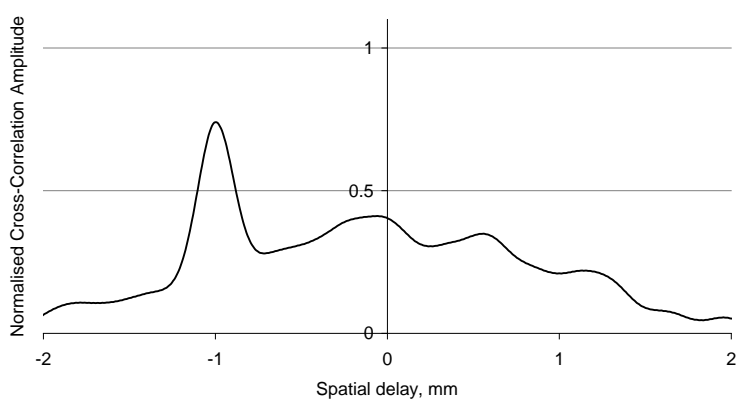

(a) $0.1^{\circ}$

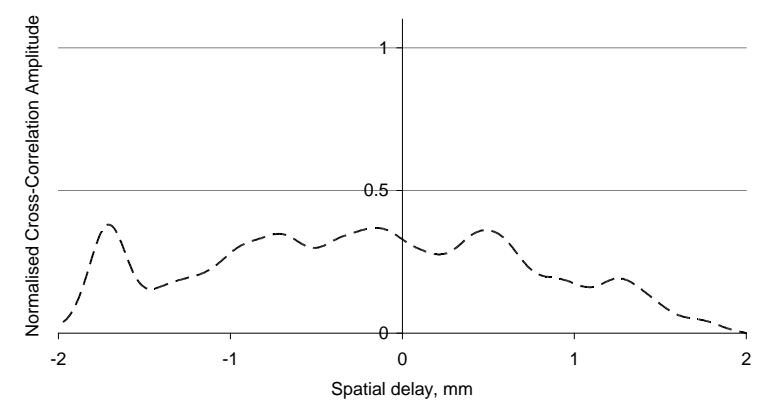

(b) $0.2^{\circ}$

Figure 4.2 Normalised cross-correlations

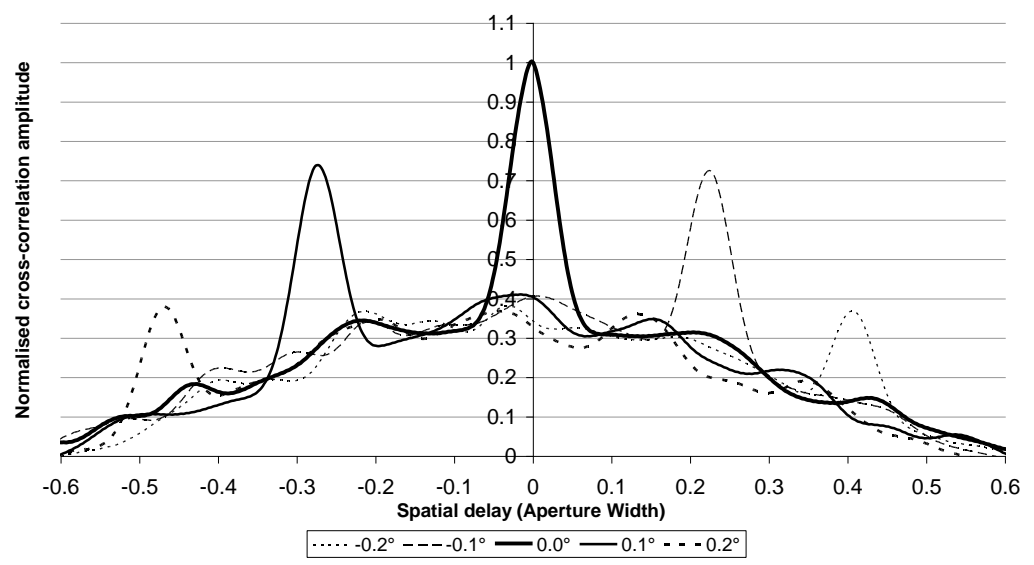

Figure 4.3: Normalised cross-correlation for tilting surface treated with Retro-Reflective Tape using a beam spot diameter of $600 \mu \mathrm{m}$ 
Figure 4.4 shows average peaks of the normalised cross-correlations, for a tilting surface, together with spatial delay and normalised cross-correlation amplitude error bars representing one standard deviation of each respectively. Parallax and backlash errors, in the displacement stage, cause measurement repeatability issues and the combination of these errors is estimated from the mean value of the standard deviation of all the spatial delays as $+/-0.03^{\circ}$. This is $30 \%$ of the increment and larger than desirable, but is the best currently achievable. Figure 4.4 shows clear evidence of the effect, on the normalised cross-correlation amplitude, of the Airy disc profile. The intensity drops to around 20-30\% of the peak intensity as the speckles translate to the boundaries of the Vibrometer aperture but the phase of the speckle should not affected by the transition. The photodetector output is the result of a phasor summation over all the collected speckles, with changes in resultant phase appearing as speckle noise. When the speckle pattern behaviour generating speckle noise is dominated by translation, the changing phase of the photodetector output is governed by new speckles entering the collected region while existing speckles leave. This appears to be the dominant feature for the surfaces without retroreflective tape but, when tape is used, the summation is affected by the relatively low amplitudes of the new speckles entering and the existing speckles leaving the region sampled. Additionally, the speckles that continue to contribute to the phasor summation change in amplitude as they translate across the Airy disc profile. The difference in the normalised cross-correlation amplitude for retro-reflective tape therefore implies that the mechanism by which speckle noise is generated when using tape is different to that with untreated surfaces. The additional changes are likely to increase speckle noise amplitudes from a taped surface compared to an untreated rough surface generating a fully developed speckle pattern and this has been observed in measured data ${ }^{[6]}$.

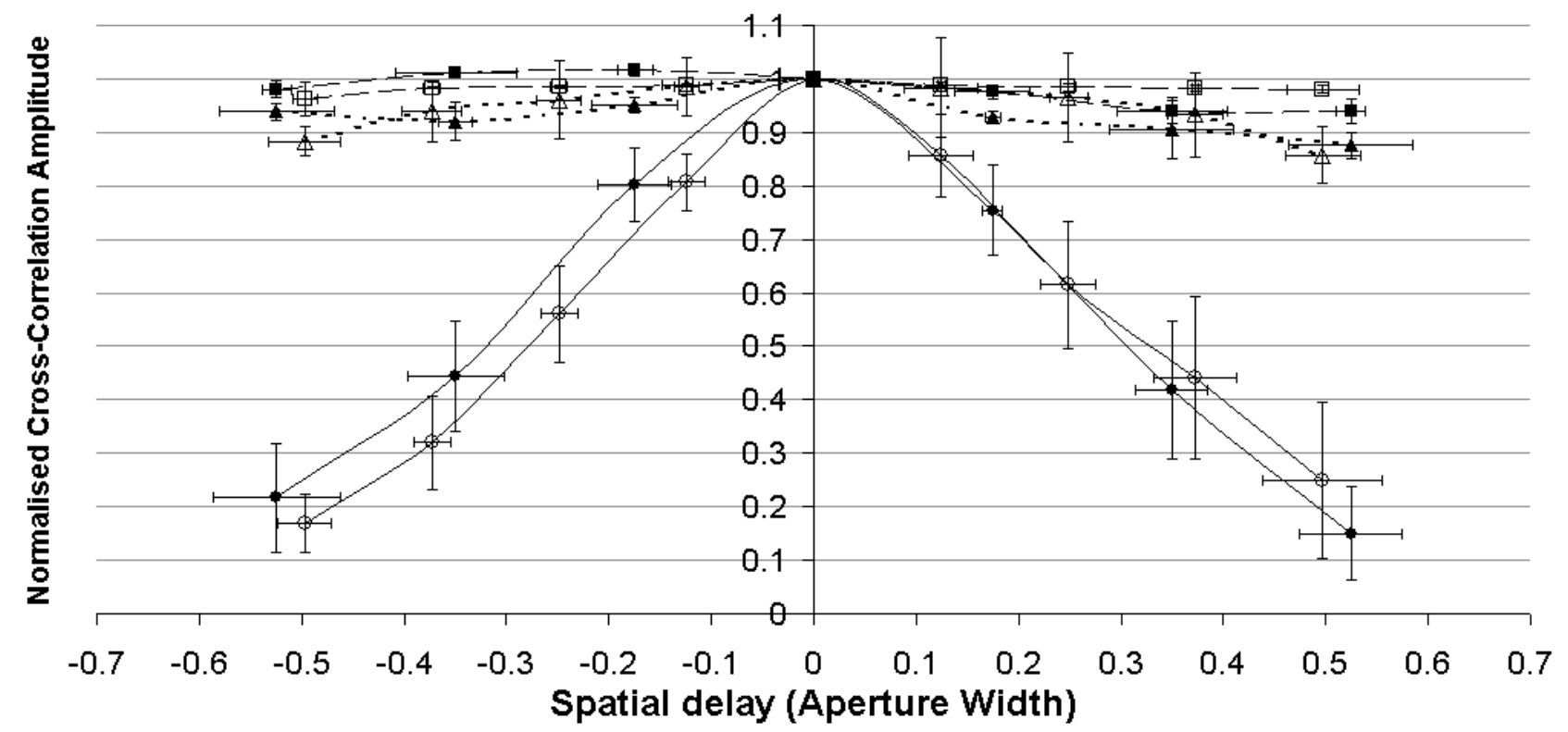

\begin{tabular}{|c|c|c|}
\hline - Retro-Reflective Tape $(100 \mu \mathrm{m})$ & $-\Delta-1.0 u m(100 \mu m)$ & $\neg-11 \mathrm{~nm}(100 \mu \mathrm{m})$ \\
\hline - - Retro-Reflective Tape $(600 \mu \mathrm{m})$ & $-\ldots-1.0 \mu \mathrm{m}(600 \mu \mathrm{m})$ & $--11 \mathrm{~nm}(600 \mu \mathrm{m})$ \\
\hline
\end{tabular}

Figure 4.4: Average normalised cross-correlation peaks of a tilting surface $+/$ - one standard deviation using beam diameters of $100 \mu \mathrm{m}$ and $600 \mu \mathrm{m}$

Successive points on the plot are separated by incremental displacements of $0.1^{\circ}$. There are more measured points across a delay equal to the aperture width when using the Vibrometer with a beam spot diameter of $100 \mu \mathrm{m}$. A smaller beam spot diameter produces large speckles and a larger aperture allows a greater spatial delay before correlation is lost. Consequently, when using the smaller beam diameter, whose Vibrometer has a larger aperture, the sampled region remains correlated over a greater angular displacement, as shown in Figure 4.4. This has been shown to reduce speckle noise levels when using a smaller beam spot diameter on a tilting surface ${ }^{[6]}$. Closer observation of the surfaces without tape show that the smooth surface $(\mathrm{Ra} 11 \mathrm{~nm})$ with the mirror-like reflection maintains its correlation amplitude higher than the rough surface $(\mathrm{Ra} 1.0 \mu \mathrm{m})$ with the speckle pattern, particularly at the extremes of the spatial delay. A possible reason for this is due to the elongation of the beam spot, described in Section 3.1. As the surface tilts there is a change to 
the population of surface scatterers and the speckles evolve. However, it can also be seen, from Figure 4.4, that this has a small effect on the correlation amplitude and it is considered to be of little practical significance. Ultimately, this process will be repeated for all the surface finishes listed in Table 1 using both beam diameters for tilt and in-plane surface motions.

When in-plane surface motions are considered, the speckle should translate comparably with the surface displacement while simultaneously evolving. This should cause the peak of the normalised cross-correlation to translate with a spatial delay equal to the surface displacement and diminish in amplitude. Figure 4.5 shows an example of the expected and actual spatial delays of the normalised cross-correlation peaks, expressed in terms of $D$, from a surface moving in-plane over a range of $D / \sqrt{2}$, with Ra $0.37 \mu \mathrm{m}$ using a beam diameter of $100 \mu \mathrm{m}$. Figure 4.5 shows that the speckles translated not only to a greater extent but also in a direction opposite to that expected.

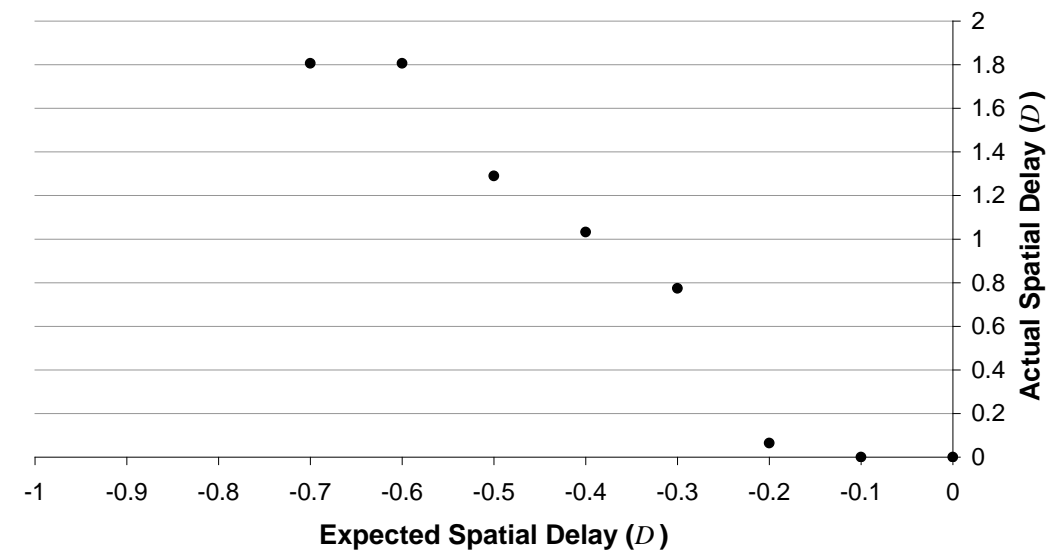

Figure 4.5: Expected spatial delays and actual spatial delays of the normalised cross-correlation peaks from a surface, moving in-plane, with Ra $0.37 \mu \mathrm{m}$ using a beam spot diameter of $100 \mu \mathrm{m}$

This unexpected behaviour is considered to be the result of the surface not being entirely flat and evidence for this can be seen in the topographical plot seen in Figure 4.6. As the surface translates, the change to the angle of the localised surface normal alters the scattering angles and the speckles will translate. A surface which is significantly convex, in the direction the surface is moving in, will cause the speckles to translate in an opposite direction to the motion of the surface. The effect of a change to the local normal to the surface is greater for a smaller beam and this effect is not evident in the speckle motions observed when using the larger beam spot diameter of $600 \mu \mathrm{m}$. The implication of this, with regard to speckle noise in Laser Vibrometry, is that the flatness of the surface can also influence speckle noise for in-plane surface motions.

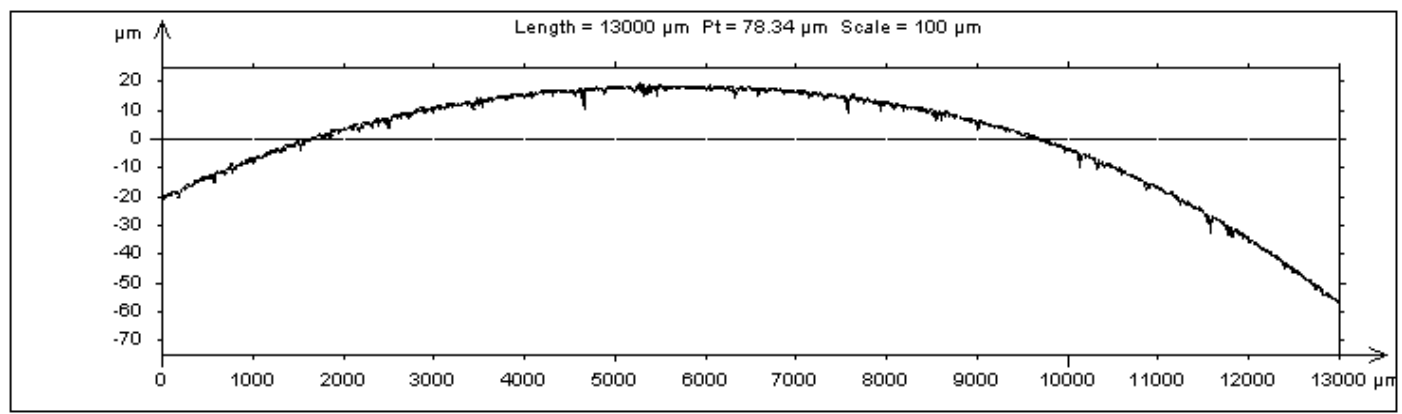

Figure 4.6: Topography results for profile of surface with roughness Ra $0.37 \mu \mathrm{m}$ 


\section{CONCLUSIONS}

Qualitative analysis of speckle images can be useful in ascertaining speckle behaviour. This paper has shown both translation and evolution qualitatively and proposed a method to analyse the speckle motions quantitatively using the cross-correlation of the high resolution images. It has shown images of speckle patterns from surfaces producing fully developed speckle patterns through to smooth surfaces producing mirror-like reflections (Ra $1.0 \mu \mathrm{m}$ to $11 \mathrm{~nm}$ ) and also speckle patterns produced from retro-reflective tape with two beam spot diameters of $100 \mu \mathrm{m}$ and $600 \mu \mathrm{m}$. When the target surface is tilting, speckles predominantly translate. However, the speckle behaviour when using retro-reflective tape exhibits a change to the amplitude in addition to the translational motion. The phase of the resultant is therefore altered by a different speckle behaviour and so the mechanism producing speckle noise when using retro-reflective tape is different to the other surfaces examined in this paper. This behaviour is likely to cause increased speckle noise levels to that which is generated by fully developed speckle patterns, from surfaces without the tape, evidence of which can be seen in measured data ${ }^{[6]}$. In comparison to the larger beam, the $100 \mu \mathrm{m}$ beam spot diameter produces larger speckles and together with a larger aperture the sampled region remains correlated for longer durations, decreasing the rate of change of the resultant phase, ultimately reducing speckle noise levels. In-plane surface motions have been shown to produce speckle behaviours which evolve and translate. The degree of decorrelation is reliant on the surface motion relative to the beam spot diameter and the translation is dependent on the surface displacement and also the local normal of the surface, particularly for the smaller beam diameter. Therefore speckle noise generated from a surface which is moving in-plane can also be influenced by the flatness of the surface. For an in-plane surface motion a greater surface displacement is required for the correlation to be lost when using a larger beam diameter. For a fixed surface displacement, a larger beam diameter therefore reduces the effects of speckle noise.

\section{Acknowledgements}

The authors would like to acknowledge the Engineering and Physical Sciences Research Council (EPSRC) for providing financial support and Lambda Photometrics Limited for their assistance with the optical profiler used in this research.

\section{References}

[1] Goodman J W, [Laser Speckle and Related Phenomena], Springer-Verlag, Berlin, pp 9-75 (1984)

[2] Tullis I and Rothberg S J, "Spatially integrated speckle intensity: maximum resistance to decorrelation caused by in-plane target displacement", Applied Optics, 37(30), 7062-7069 (1998)

[3] Asakura T and Takai N, "Dynamic Laser Speckle and Their Application to Velocity Measurements of the Diffuse Object", Appl. Phys., 25, 179-194 (1981)

[4] Jakeman E and Ridley K D, [Modelling Fluctuations in Scattered Waves], Taylor \& Francis, pp 7 \& 8 (2006)

[5] Goodman J W, "Speckle with a finite number of steps", Applied Optics, 47(4), 111-118 (2008)

[6] Martin P and Rothberg S J, "Introducing Speckle Noise maps for Laser Vibrometry", Optics and Lasers in Engineering, submitted July 2007.

[7] Rothberg S J, Baker J R and Halliwell N A, "Laser Vibrometry: Pseudo-vibrations", Journal of Sound and Vibration, 135(3), 516-522 (1989) 